\title{
Author Correction: Cdkn1a deletion improves stem cell function and lifespan of mice with dysfunctional telomeres without accelerating cancer formation
}

Aaheli Roy Choudhury, Zhenyu Ju, Meta W. Djojosubroto, Andrea Schienke, Andre Lechel, Sonja Schaetzlein, Hong Jiang, Anna Stepczynska, Chunfang Wang, Jan Buer, Han-Woong Lee, Thomas von Zglinicki, Arnold Ganser, Peter Schirmacher, Hiromitsu Nakauchi and K. Lenhard Rudolph

Correction to: Nature Genetics https://doi.org/10.1038/ng1937, published online 3 December 2006.

In the version of this article initially published, Supplementary Figs. $2 \mathrm{~d}$ and $4 \mathrm{~b}$ contained errors in the representative photographs of organs and cells. In Supplementary Fig. 2d, in the representative photographs of hematoxylin- and eosin-stained longitudinal sections of the spleen in 4-month-old mice of the indicated genotypes, the original photograph for the cohort of iG4 mice showed a spleen sample from a different genotype and age cohort (iF1, Cdkn1 $a^{-/-}, 12-15$ months), which was correctly displayed in Fig. 1d. The corrected Supplementary Fig. 2d contains new representative photographs of the spleens of 4-month-old mice of the indicated genotypes. In Supplementary Fig. $4 \mathrm{~b}$, in the representative photographs of SA- $\beta$ Gal staining of hematopoietic stem and progenitor cells (KSL cells) of 12- to 15-month-old iF1 and iG4 mice in the bottom row, the original photographs showed a partially overlapping area of cells from the same image. The corrected Supplementary Fig. 4 b contains new representative photographs of KSL cells from 4-month-old mice of the indicated genotypes. The errors have been corrected in the revised Supplementary Figs. 2 and 4 included with this Author Correction online.

\section{Additional information}

Supplementary information is available for this paper at https://doi.org/10.1038/s41588-020-0593-6.

Published online: 30 April 2020

https://doi.org/10.1038/s41588-020-0593-6

(C) The Author(s), under exclusive licence to Springer Nature America, Inc. 2020 